\title{
Eliminación del plomo por curado casero
}

Luisa Torres-Sánchez, M. en C.,1) Lizbeth López-C arrillo, Ph.D., ${ }^{(2)}$ Camilo Ríos, Ph.D.(3)

\section{Torres-Sánchez L, López-Carrillo L, Ríos C. Eliminación del plomo por curado casero. Salud Publica Mex 1999;41 supl 2:S106-S108.}

\section{Resumen}

Objetivo. Evaluar el curado ácido como medida preventiva para reducir el contenido de plomo en vasijas de barro vidriadas. Material y métodos En 27 vasijas de barro procedentes de cuatro estados de México se determinó, mediante espectrofo to metría de absorción atómica, el nivel de plomo residual, después de cuatro lavados con ácido acético al 3\%. Resultados El contenido de plomo en el ácido acético utilizado para lavar las vasijas disminuyó proporcio nalmente con el número de lavados, aunque se conservó por arriba de los niveles permisibles (2.5-7.0 p.p.m.). Conclusiones El curado ácido de piezas de barro no es una medida preventiva útil para reducir la exposición a plomo.

Palabras clave: plomo; curado ácido; prevención primaria; cerámica vidriada; México
Torres-Sánchez L, López-Carrillo L, Ríos C. Lead elimination by traditional acidic curing. Salud Pblica Mex 1999;41 suppl 2:S106-S108.

\section{A bstract}

Objective.To evaluate traditional acidic curing as a preventive method for reducing lead content of glazed ceramic ware. Material and methods. In 27 ceramic ware pieces from four states in Mexico, the level of residual lead was determined through atomic absorption spectrophotometry after four washings with acetic acid 3\%. Results. The lead content of the vinegar washing diminished proportionally with the number of washings, although it remained highly above the permissible levels of this metal (2.5-7.0 p.p.m.). Conclusions. Traditional acidic curing of glazed ceramic ware is not a useful preventive measure for reducing lead exposure.

Key words: lead; traditional curing; primary prevention; lead glazed ceramic; Mexico
$E$ $\mathrm{n}$ diversos estudios se ha identificado el uso de cerámica vidriada para cocinar y almacenar alimentos como una de las fuentes más importantes de exposición a plomo, que determinan el nivel de dicho metal en sangre en la población mexicana. ${ }^{1}$

Además de las intoxicaciones agudas que pueden ocurrir por la ingestión de plomo, se ha documentado que la exposición crónica al mismo puede ocasionar daño neuroconductual en menores de nueve años de edad ${ }^{2,3} \mathrm{y}$, entre las mujeres gestantes, es muy posible que origine trastornos como el aborto y la prematurez, entre otros. ${ }^{4,5}$

Ante esta situación, la normatividad mexicana ha planteado una reducción de los niveles máximos permisibles de plomo en el vidriado de las piezas de cerámica. ${ }^{6,7}$ En noviembre de 1993 se publicó una norma específica mediante la cual se establece que a partir de esa fecha se llevará a cabo un proceso gradual de

(1) Centro de Investigación en Sistemas de Salud, Instituto N acional de Salud Pública (IN SP), México.

(2) Centro de Investigación en Salud Poblacional, IN SP, México.

(3) Instituto $\mathrm{N}$ acional de $\mathrm{N}$ eurología y N eurocirugía, México.

Fecha de recibido: 13 de mayo de 1998 - Fecha de aprobado: 7 de abril de 1999

Solicitud de sobretiros: Dra. Lizbeth López Carrillo. Instituto N acional de Salud Pública. Universidad 655 colonia Santa María A huacatitlán, 62508 Cuernavaca, Morelos, México.

Correo electrónico: lizbeth@ insp3.insp.mx 
disminución del contenido de plomo liberado en medio ácido por las piezas de cerámica hasta niveles de 2.5 a 7.0 p.p.m., dependiendo del tamaño y forma de la pieza. ${ }^{7}$

Por otra parte, antes de utilizarlas los usuarios de vasijas de barro comúnmente llevan a cabo el curado casero de las mismas con objeto de reducir la permeabilidad de sus paredes y hacerlas menos quebradizas. ${ }^{8}$

El objetivo de este trabajo fue evaluar si el curado ácido a base de vinagre comercial (ácido acético al 3\%) podría reducir el contenido de plomo en vasijas de barro vidriadas, hasta los niveles máximos permisibles establecidos en la legislación mexicana vigente.

\section{Material y métodos}

Durante 1995 se seleccionaron 27 vasijas de barro vidriado procedentes de los estados de México, Oaxaca, Puebla, y Tlaxcala; todas se fabricaron con un proceso de laqueado similar.

Específicamente, se obtuvieron tres vasijas fabricadas en el pueblo de Metepec y una en el pueblo de Tecomatepec (Estado de México); dos de Sta. Ma. Atzompa (Oaxaca), nueve de las siguientes localidades de Puebla: San Jerónimo Ocotitlán (1), Amozoc (3), Barrio de la Luz (2), San Miguel Tenextatiloya (1), Chignahuapan (1), Aquixtla (1), y 12 vasijas procedentes de las poblaciones del estado de Tlaxcala que a continuación se relacionan: San Salvador Tzompantepec (4), Espanita (3) y la Trinidad Teneyecac (5).

Con el objetivo de reproducir el curado casero con vinagre, a cada vasija se le añadió ácido acético al 3\% glacial, de grado analítico, E. Merck (México). El contenido de plomo de esta solución fue analizado previo a su uso y se encontró por abajo del límite de detección del método.

Las adiciones de ácido acético en cada pieza se hicieron durante cuatro días consecutivos, dejándolo reposar a temperatura ambiente por un lapso de 24 horas en cada oportunidad. Las concentraciones de plomo en el ácido acético se determinaron mediante espectrofotometría de absorción atómica con horno de grafito (Perkin Elmer HGA-2200). El control de calidad del procedimiento se realizó internamente con estándares de plomo procedentes del Centro para el Control y la Prevención de Enfermedades, de Atlanta, Estados Unidos (CDC).

La precisión del método de análisis fue del $8 \%$ (coeficiente de variación porcentual) para el análisis entre días. La exactitud se garantizó con el análisis de tres muestras de sangre de bovino, cuyos valores de concentración se encontraron en un intervalo de $\pm 2 \mathrm{DE}$ en relación con el valor que definió el CDC de Atlanta.
Los niveles medios de plomo que se obtuvieron en cada lavado se compararon con los resultantes del primer lavado, mediante análisis de varianza (ANOVA) y utilizando el paquete estadístico STATA 4.0.

\section{Resultados}

Los niveles medios de plomo en cada día de lavado de acuerdo al estado de procedencia de la vasija de barro se muestran en el cuadro I. En general el contenido medio de plomo en el ácido acético disminuyó proporcionalmente con el número de lavados, de tal forma que en el ácido acético utilizado en el cuarto lavado los niveles medios de plomo fueron menores en comparación con los encontrados en el primer lavado, excepto para las vasijas fabricadas en el estado de Tlaxcala. Asimismo, se observa que las vasijas procedentes del estado de Oaxaca liberaron la mayor cantidad de plomo (4239.90 p.p.m).

Sin duda el hallazgo más importante es el hecho de que, después de cuatro lavados el contenido de plomo en el ácido acético analizado era 104.82 veces superior a las 2.5 a 7 p.p.m. que establece la norma en vigor, para el total de las piezas. Sin embargo, el ácido acético que se empleó en el lavado de las piezas provenientes del estado de Oaxaca llegó a tener un exceso de plomo casi 150 veces superior a lo que fija la norma.

\section{Discusión}

De acuerdo con los resultados de este estudio, el plomo contenido en el vidriado de las cazuelas de barro

\section{Cuadro 1}

MedidAs geométricas del CONTENIDO de PLomo (P.P.M.) EN EL ÁCIDO ACÉTICO SEGÚN días de LAVAdo.* Méxıco 1995

\begin{tabular}{|c|c|c|c|c|c|c|}
\hline \multirow[b]{2}{*}{$\begin{array}{l}\text { Estados de } \\
\text { procedencia }\end{array}$} & \multirow[b]{2}{*}{ (n) } & \multicolumn{4}{|c|}{ Días de lavado } & \multirow[b]{2}{*}{$\begin{array}{l}\text { Exceso de } \\
\text { plomo }\end{array}$} \\
\hline & & 1 & 2 & 3 & 4 & \\
\hline axaca & (2) & 4239.90 & 1913.32 & 1941.87 & 1037.75 & 148.25 \\
\hline uebla & (9) & 731.06 & 905.61 & 841.06 & 640.49 & 91.50 \\
\hline léxico & (4) & $663.38^{\S}$ & 327.86 & 244.73 & 560.18 & 80.02 \\
\hline laxcala & (12) & 668.35 & $258.39 \#$ & 2237.87 & 849.34 & 121.33 \\
\hline otal & (27) & 788.75 & 471.59 & 1151.36 & 733.74 & 104.82 \\
\hline \multicolumn{7}{|c|}{$\begin{array}{l}\text { * Ac. acético al } 3 \% \\
\text { ₹azón exceso de plomo= Plomo observado/Plomo permitido: con base } \\
\text { en el resultado del cuarto lavado con vinagre } \\
\text { \$ } p<0.05 \text { Estado de México vs } 0 \text { axaca } \\
\# p<0.05 \text { en comparación con el tercer día }\end{array}$} \\
\hline
\end{tabular}


no sería liberado totalmente mediante un curado ácido a base de vinagre comercial durante cuatro días.

Los niveles promedio de plomo identificados en el ácido acético que se utilizó en el lavado de las diferentes vasijas analizadas, coinciden con los que encontraron Viniegra y colaboradores, ${ }^{9}$ y que fueron similares en piezas de barro vidriado procedentes de los estados de Guanajuato, Oaxaca, Puebla y Jalisco.

De manera contraria a lo esperado, las vasijas de barro procedentes del estado de Tlaxcala liberaron mayores niveles de plomo durante el tercer y cuarto lavado. Lo anterior pudo deberse a un daño importante en el esmalte durante el proceso de lavado con una sustancia ácida. ${ }^{8}$

De estos hallazgos surgen al menos dos interpretaciones en materia de salud pública: en primer lugar, que es nula la posibilidad de eliminar la exposición a plomo de las vasijas de loza vidriada si se utilizan medios caseros ácidos. Al no haberse logrado una disminución importante de los niveles de plomo usando el ácido acético, considerado como un medio óptimo para solubilizar los iones de plomo que no llegaron a fundirse por falta de la temperatura de cocción adecuada $\left(990^{\circ} \mathrm{C}\right){ }_{,}^{8}$ sería necesario evaluar la capacidad de otros tipos de curados -como el ajo, la manteca, o bien la acción de hervir en las ollas una solución jabonosa, para eliminar la exposición a plomo.

En segundo lugar, los alimentos preservados en vinagre, tales como chiles, vegetales, etc., no deben ser almacenados en recipientes de cerámica vidriada, ya que los niveles de plomo que pueden ser solubilizados en el vinagre de los mismos alcanzarían niveles por demás elevados. En este sentido baste con tomar en cuenta que el contenido de plomo liberado por el vidriado de las vasijas procedentes del estado de Oaxaca, superó las 4000 p.p.m. Se trata aquí de una medida de prevención que tendría que observar la población general mientras se sigan utilizando este tipo de vasijas. Además, se justifica un sistema de vigilancia epidemiológica relacionado con los niveles de plomo presentes en el barniz de las ollas de barro vidriado, con la finalidad de evaluar el cumplimiento de la normatividad.

Por último y no obstante los esfuerzos que se han realizado en materia de normatividad, de investigación y búsqueda de acciones preventivas, el uso de la cerámica vidriada es parte de la cultura nacional, constituye un rasgo cultural y, sobre todo, una fuente importante de trabajo para muchos mexicanos, por lo que encontrar un justo balance para eliminar esta fuente de exposición a plomo constituye un compromiso que han manifestado las autoridades gubernamentales y que requerirá de la aceptación y la respuesta activa de un sector importante de la población: los usuarios mismos de vasijas de barro.

\section{Referencias}

1. López-C arrillo L,Torres-Sánchez L, G arrido F, Papaqui-Hernández J, Palazuelos-Rendón E, López-Cervantes M. Prevalence and determinants of lead intoxication in Mexican children of low socioeconomic status. Environ Health Perspect 1996;104:1208-1211.

2. Muñoz I, Romieu I, Palazuelos E, Mancilla-SánchezT, Meneses $G$ onzález F, Hernández-Avila M. Blood lead level and neurobehavioral development among children living in M exico C ity.A rch Environ Health 1993;48: 132-139.

3. Bellinger $D$, Levinton $A, W$ aternaux $C, N$ eedledman $H$, Rabinowitz $M$. Longitudinal analyses of prenatal and postnatal lead exposure and early cognitive development. N Engl J Med 1987;316:1037-1043.

4. Davis JM, Svendsgaard DJ. Lead and child development. Nature 1987;329:297-300.

5. Andrews KW, Savitz DA, H ertz-Picciotto IH. Prenatal lead exposure in relation to gestational age and birth weigth:A review of epidemiological studies. Am J Ind Med 1994;26:13-32.

6. Secretaría de Salud. N O M-010-SSA 1-1993. N ormas y anteproyectos de normas oficiales mexicanas, 12/11/93, 1993.

7. Secretaría de Salud. N O M-011-SSA 1-1993. N ormas y anteproyectos de normas oficiales mexicanas, 12/11/93, 1993.

8. Huerta-Cendejas S, Díaz-G onzález A. El problema de la toxicidad en la loza artesanal mexicana. Salud Publica Mex 1974;26:83-88.

9.Viniegra G, Escobar R. El riesgo del saturnismo por la cerámica folklórica mexicana. Salud Publica Mex 1966;7:69-77. 